\title{
Beauville surfaces with abelian Beauville group
}

\author{
Gabino González-Diez Gareth A. Jones \\ David Torres-Teigell
}

October 22, 2018

\begin{abstract}
A Beauville surface is a rigid surface of general type arising as a quotient of a product of curves $C_{1}, C_{2}$ of genera $g_{1}, g_{2} \geq 2$ by the free action of a finite group $G$. In this paper we study those Beauville surfaces for which $G$ is abelian (so that $G \cong \mathbb{Z}_{n}^{2}$ with $\operatorname{gcd}(n, 6)=1$ by a result of Catanese). For each such $n$ we are able to describe all such surfaces, give a formula for the number of their isomorphism classes and identify their possible automorphism groups. This explicit description also allows us to observe that such surfaces are all defined over $\mathbb{Q}$.
\end{abstract}

2010 Mathematics Subject Classification: 14J50 (primary); 14J25 14J29 20B25 (secondary)

\section{Introduction and statement of results}

A complex surface $S$ is said to be isogenous to a product if it is isomorphic to the quotient of a product of curves $C_{1} \times C_{2}$ of genus $g_{1}=g\left(C_{1}\right), g_{2}=g\left(C_{2}\right) \geq 1$ by the free action of a finite subgroup $G$ of $\operatorname{Aut}\left(C_{1} \times C_{2}\right)$, the automorphism group of $C_{1} \times C_{2}$.

If additionally the curves satisfy $g_{1}, g_{2} \geq 2$ we say that $S$ is isogenous to a higher product. We will always assume that $g_{1} \leq g_{2}$.

It is not difficult to see that an element of $\operatorname{Aut}\left(C_{1} \times C_{2}\right)$ either preserves each curve or interchanges them, the latter being possible only if $C_{1} \cong C_{2}$. Consequently one says that $S$ is of unmixed type if no element of $G$ interchanges factors, and of mixed type otherwise.

On the other hand if two elements $g, h \in G$ interchange factors it is clear that $g h$ belongs to the subgroup $G^{0}<G$ of factor-preserving elements, and therefore $\left[G: G^{0}\right]=2$.

There is always a minimal realization of $S$ in the sense that $G^{0}$ acts faithfully on each factor $C_{i}$. This is because if for instance $G^{0}$ did not act faithfully on $C_{1}$, so that there exists a subgroup $G^{\prime} \triangleleft G$ acting trivially on $C_{1}$, then we could write

$$
S \cong \frac{C_{1} \times C_{2}}{G}=\frac{C_{1} / G^{\prime} \times C_{2} / G^{\prime}}{G / G^{\prime}}=\frac{C_{1} \times\left(C_{2} / G^{\prime}\right)}{G / G^{\prime}}
$$


Hence, from now on we will always assume that the realization $S \cong\left(C_{1} \times C_{2}\right) / G$ is minimal.

A Beauville surfaces is a particular type of surface isogenous to a product. These surfaces were introduced by Catanese in [6] following a construction by Beauville in [5] (see Example 1 below), and they have been subsequently studied by himself, Bauer and Grunewald ([1], 2], 4]), somewhat later by Fuertes, González-Diez and Jaikin ([8], [9]), and more recently by Jones, Penegini, Garion, Larsen, Lubotzky, Guralnick, Malle, Fairbairn, Magaard and Parker ([10, [14, [1], [12, [13, 7]).

A Beauville surface is a compact complex surface $S$ isogenous to a higher product, $S \cong\left(C_{1} \times C_{2}\right) / G$, where $G^{0}$ acts on each of the curves in such a way that $C_{1} / G^{0} \cong \mathbb{P}^{1}, C_{2} / G^{0} \cong \mathbb{P}^{1}$, and the natural projections $C_{1} \longrightarrow \mathbb{P}^{1}$ and $C_{2} \longrightarrow \mathbb{P}^{1}$ ramify over three values.

Catanese proved that both the group $G$ and the curves $C_{1}, C_{2}$ - hence also the genera $g_{1}, g_{2}$ - are invariants of the Beauville surface. Consequently we will say that the surface $S$ has (Beauville) group $G$ and covering product $C_{1} \times C_{2}$.

Here we will consider only unmixed Beauville surfaces. In this case there exist isomorphisms $\varphi_{i}: G \longrightarrow H_{i} \leq \operatorname{Aut}\left(C_{i}\right)$ so that the action of an element $g \in G$ on a point $\left(p_{1}, p_{2}\right) \in C_{1} \times C_{2}$ is given by $g\left(p_{1}, p_{2}\right)=\left(\varphi_{1}(g)\left(p_{1}\right), \varphi_{2}(g)\left(p_{2}\right)\right)$. From this point of view giving an unmixed Beauville surface amounts to specifying an isomorphism $\phi=\varphi_{2} \circ \varphi_{1}^{-1}$ between groups $H_{1} \leq \operatorname{Aut}\left(C_{1}\right)$ and $H_{2} \leq$ $\operatorname{Aut}\left(C_{2}\right)$ satisfying the following two conditions

(C1) $C_{i} / H_{i}$ is an orbifold of genus zero with three branching values, and

(C2) for any non-identity element $h_{1} \in H_{1}$ which fixes a point on $C_{1}$ the element $h_{2}=\phi\left(h_{1}\right) \in H_{2}$ acts freely on $C_{2}$ (so that the action $h\left(p_{1}, p_{2}\right)=$ $\left(h_{1}\left(p_{1}\right), h_{2}\left(p_{2}\right)\right)$ is free on $\left.C_{1} \times C_{2}\right)$.

It turns out that the problem of deciding whether a finite group is an unmixed Beauville group is purely group theoretical [6]. This occurs if and only if $G$ admits two triples of generators $\left(a_{1}, b_{1}, c_{1}\right)$ and $\left(a_{2}, b_{2}, c_{2}\right)$ such that

(i) $a_{1} b_{1} c_{1}=a_{2} b_{2} c_{2}=1$;

(ii) $\frac{1}{\operatorname{ord}\left(a_{i}\right)}+\frac{1}{\operatorname{ord}\left(b_{i}\right)}+\frac{1}{\operatorname{ord}\left(c_{i}\right)}<1, i=1,2$;

(iii) $\Sigma\left(a_{1}, b_{1}, c_{1}\right) \cap \Sigma\left(a_{2}, b_{2}, c_{2}\right)=\left\{\operatorname{Id}_{G}\right\}$, where we define

$$
\Sigma(a, b, c)=\left\{\bigcup_{g \in G}\left(g\langle a\rangle g^{-1} \cup g\langle b\rangle g^{-1} \cup g\langle c\rangle g^{-1}\right)\right\}
$$

This is a very useful characterization since it allows the use of computer programs such as GAP or MAGMA to solve the problem for groups of low order. 
The first example of a Beauville surface was given by A. Beauville some twenty years before the term was coined by Catanese. It appears as an exercise at the end of his book on complex surfaces [5] as an example of a complex surface of general type whose two geometrical invariants $q$ and $p_{g}$ vanish.

Example 1 (Beauville). Let us denote by $\mathbb{Z}_{n}=\mathbb{Z} / n \mathbb{Z}$ the group of integers modulo $n$ and by $F_{n}$ the Fermat curve of degree $n$,

$$
F_{n}=\left\{[x: y: z] \in \mathbb{P}^{2}(\mathbb{C}): x^{n}+y^{n}+z^{n}=0\right\} .
$$

For $n=5$ the group $G=\mathbb{Z}_{5}^{2}$ acts freely on $F_{5} \times F_{5}$ in the following way: for each $(\alpha, \beta) \in G$ we put

$$
(\alpha, \beta)\left(\left[\begin{array}{l}
x_{1} \\
y_{1} \\
z_{1}
\end{array}\right],\left[\begin{array}{l}
x_{2} \\
y_{2} \\
z_{2}
\end{array}\right]\right)=\left(\left[\begin{array}{c}
\xi^{\alpha} x_{1} \\
\xi^{\beta} y_{1} \\
z_{1}
\end{array}\right],\left[\begin{array}{c}
\xi^{\alpha+3 \beta} x_{2} \\
\xi^{2 \alpha+4 \beta} y_{2} \\
z_{2}
\end{array}\right]\right)
$$

where $\xi=e^{2 \pi i / 5}$. It is easy to see that the surface $S:=\left(F_{5} \times F_{5}\right) / \mathbb{Z}_{5}^{2}$ is an unmixed Beauville surface.

Obviously the formula

$$
(\alpha, \beta)([x: y: z])=\left[\xi^{\alpha} x: \xi^{\beta} y: z\right] \quad\left(\xi=e^{2 \pi i / n}\right)
$$

also defines an action of the group $\mathbb{Z}_{n}^{2}$ on $F_{n}$ when $n>5$. The quotient $F_{n} / \mathbb{Z}_{n}^{2}$ is always an orbifold of genus zero with three branching values, all of them of order $n$. Therefore any group automorphism $\phi: \mathbb{Z}_{n}^{2} \longrightarrow \mathbb{Z}_{n}^{2}$ satisfying condition $(\mathrm{C} 2)$ above gives rise to a Beauville surface, something that can occur only when $\operatorname{gcd}(n, 6)=1($ see [6]). Since any such $\phi$ is necessarily of the form

$$
\phi=\phi_{A}:\left(\begin{array}{c}
\alpha \\
\beta
\end{array}\right) \longmapsto A \cdot\left(\begin{array}{c}
\alpha \\
\beta
\end{array}\right) \quad \text { for some } \quad A=\left(\begin{array}{cc}
a & b \\
c & d
\end{array}\right) \in \mathrm{GL}_{2}\left(\mathbb{Z}_{n}\right),
$$

any surface obtained in this way is of the form $S_{A}^{n}=\left(F_{n} \times F_{n}\right) / G_{A}$, where $G_{A}$ is the subgroup of $\operatorname{Aut}\left(F_{n} \times F_{n}\right)$ defined by

$$
G_{A}=\left\{\left(\left(\begin{array}{c}
\alpha \\
\beta
\end{array}\right), A\left(\begin{array}{c}
\alpha \\
\beta
\end{array}\right)\right):\left(\begin{array}{c}
\alpha \\
\beta
\end{array}\right) \in G\right\} \cong G
$$

In this way the action of an element $(\alpha, \beta) \in \mathbb{Z}_{n}^{2}$ on $F_{n} \times F_{n}$ is given by

$$
(\alpha, \beta)\left(\left[\begin{array}{l}
x_{1} \\
y_{1} \\
z_{1}
\end{array}\right],\left[\begin{array}{l}
x_{2} \\
y_{2} \\
z_{2}
\end{array}\right]\right)=\left(\left[\begin{array}{c}
\xi^{\alpha} x_{1} \\
\xi^{\beta} y_{1} \\
z_{1}
\end{array}\right],\left[\begin{array}{c}
\xi^{a \alpha+b \beta} x_{2} \\
\xi^{c \alpha+d \beta} y_{2} \\
z_{2}
\end{array}\right]\right) .
$$

Thus the surface constructed in Example 1 is $S_{A}^{5}$ for $A=\left(\begin{array}{ll}1 & 3 \\ 2 & 4\end{array}\right)$.

It is known that, even though in the definition of a Beauville surface both curves $C_{1}$ and $C_{2}$ are only required to have genus greater than 1 , in fact both genera have to be greater than 5 (see [9]). Thus Beauville's example $S_{A}^{5}$ above reaches the minimum possible bigenus $\left(g_{1}, g_{2}\right)=(6,6)$.

In this paper we prove the following facts: 
1. Each Beauville surface with an abelian group $G$ is isomorphic to one of the form $S_{A}^{n}$ (Theorem 1), and is defined over $\mathbb{Q}$ (Corollary 1).

2. The number $\Theta(n)$ of isomorphism classes of Beauville surfaces which have Beauville group $\mathbb{Z}_{n}^{2}$ (where $\operatorname{gcd}(n, 6)=1$ ) is given by the formula in Theorem 2. One consequence is that asymptotically $\Theta(n) \sim n^{4} / 72$ for prime powers $n$. Another is that $\Theta(5)=1$, which means that Beauville's original surface $S_{A}^{5}$ mentioned above is the only Beauville surface with group $\mathbb{Z}_{5}^{2}$.

3. The automorphism group of $S_{A}^{n}$ is either $\mathbb{Z}_{n}^{2}$, or an extension of $\mathbb{Z}_{n}^{2}$ by one of the cyclic groups $\mathbb{Z}_{2}, \mathbb{Z}_{3}$ and $\mathbb{Z}_{6}$ or by the symmetric group $\mathcal{S}_{3}$ (Proposition 1).

In 2 Bauer, Catanese and Grunewald have given a lower bound for the asymptotic behaviour of $\Theta(n)$ as $n \rightarrow \infty$. More recently, Garion and Penegini in 12 have given upper and lower bounds for $\Theta(n)$ for each $n$, and in Theorem 2 we extend their results by giving an explicit formula for $\Theta(n)$.

\section{Unmixed Beauville structures on the product of Fermat curves}

It was proved in [6] that if $S=\left(C_{1} \times C_{2}\right) / G$ is a Beauville surface with abelian group $G$ then $G=\mathbb{Z}_{n}^{2}$ with $\operatorname{gcd}(n, 6)=1$, and in 9 that in that case $C_{1}=$ $C_{2}=F_{n}$, the Fermat curve of degree $n$. Combining these two facts one gets the following slightly more precise result:

Theorem 1. Any Beauville surface with abelian Beauville group is isomorphic to one of the form $S_{A}^{n}=\left(F_{n} \times F_{n}\right) / G_{A}$.

Proof. In view of the results in [6] and 9] mentioned above it is sufficient to observe that $\operatorname{Aut}\left(F_{n}\right)$ possesses a unique subgroup isomorphic to $Z_{n}^{2}$ satisfying condition $(\mathrm{C} 1)$ in our definition of Beauville surface, and therefore any Beauville surface with group $\mathbb{Z}_{n}^{2}$ is determined by an isomorphism $A: \mathbb{Z}_{n}^{2} \longrightarrow \mathbb{Z}_{n}^{2}$ satisfying condition (C2).

This explicit description of Beauville surfaces with abelian group yields the following:

Corollary 1. Each Beauville surface with abelian group is defined over $\mathbb{Q}$.

Proof. Since the curves $F_{n}$ are obviously defined over $\mathbb{Q}$ we only have to show that so is the group $G_{A}$. That is, we have to show that the obvious action of the absolute Galois group $\operatorname{Gal}(\overline{\mathbb{Q}} / \mathbb{Q})$ on automorphisms of $F_{n} \times F_{n}$ leaves the group $G_{A}$ setwise invariant.

Now, let $\sigma$ be an arbitrary element of $\operatorname{Gal}(\overline{\mathbb{Q}} / \mathbb{Q})$, and suppose that $\sigma(\xi)=\xi^{j}$; then, clearly, $\sigma$ transforms the action of an element $(\alpha, \beta)$ of $G_{A}$ into the action of another element of $G_{A}$, namely $(j \alpha, j \beta)$. 
Remark 1. In regard to the fields of definition of quotient varieties by abelian groups we draw the reader's attention to the interesting Corollary 1.9 in the article [3] by Bauer and Catanese.

We would like to find which matrices $A=\left(\begin{array}{ll}a & b \\ c & d\end{array}\right) \in \mathrm{GL}_{2}\left(\mathbb{Z}_{n}\right)$ define Beauville surfaces $S_{A}^{n}$, that is, we would like to characterize those matrices $A$ such that the group $G_{A}$ defined above acts freely on $F_{n} \times F_{n}$. Note that the Beauville surface $S_{A}^{n}$ corresponds, in terms of triples of generators, to a pair of ordered triples satisfying conditions (i) to (iii) where, without loss, we can take the first triple to be the standard triple $((1,0),(0,1),(-1,-1))$. Then the second triple is $((a, c),(b, d),(-a-b,-c-d))$, obtained from the first one by the automorphism represented by the matrix $A \in \mathrm{GL}_{2}\left(\mathbb{Z}_{n}\right)$.

With the previous notation, the elements $(\alpha, \beta)$ fixing points in the first component are precisely those of the form $(k, 0),(0, k)$ and $(k, k)$. Equivalently the elements $(\alpha, \beta)$ that fix points in the second curve are those such that $a \alpha+b \beta=0, c \alpha+d \beta=0$ or $a \alpha+b \beta=c \alpha+d \beta$. We obtain the following:

Lemma 1. Let $A=\left(\begin{array}{ll}a & b \\ c & d\end{array}\right) \in \mathrm{GL}_{2}\left(\mathbb{Z}_{n}\right)$. The group $G_{A}$ defined above acts freely on the product $F_{n} \times F_{n}$ if and only if the following conditions hold:

$$
a, b, c, d, a+b, c+d, a-c, b-d, a+b-c-d \in U\left(\mathbb{Z}_{n}\right)
$$

where $U\left(\mathbb{Z}_{n}\right)$ is the group of units of $\mathbb{Z}_{n}$.

Proof. Let $(\alpha, \beta) \in \mathbb{Z}_{n}$ be an element fixing some point of the first curve.

If $(\alpha, \beta)=(k, 0)$ the action on the second curve is given by $(a k, c k)$, which fixes no point if and only if $a k \neq 0, c k \neq 0, a k \neq c k$. This will be true for all $k$ if and only if $a, c, a-c \in U\left(\mathbb{Z}_{n}\right)$.

Arguing the same way with elements of the form $(\alpha, \beta)=(0, k)$ and $(\alpha, \beta)=$ $(k, k)$ one obtains the result.

Note that from this lemma we can deduce the already mentioned fact, due to Catanese, that $\operatorname{gcd}(n, 6)$ must be 1 for $\mathbb{Z}_{n}^{2}$ to admit a Beauville structure.

If $n$ is even, the conditions $a, b, a+b \in U\left(\mathbb{Z}_{n}\right)$ cannot hold simultaneously. On the other hand if $n$ is a multiple of 3 then necessarily $a \equiv b \bmod 3$ and $c \equiv d \bmod 3$, but then the matrix $A$ is not invertible.

We will denote by $\mathfrak{F}_{n}$ the set of matrices in $\mathrm{GL}_{2}\left(\mathbb{Z}_{n}\right)$ satisfying the conditions in (10), and will write $e_{(\alpha, \beta)}^{A}$ for the element of $G_{A}$ corresponding to $(\alpha, \beta) \in \mathbb{Z}_{n}^{2}$. In $\mathbb{Z}_{5}^{2}$, for instance, there are 24 different matrices satisfying these conditions.

It follows from Theorem 1 that $\operatorname{Aut}\left(F_{n} \times F_{n}\right)$ acts on the set of groups $\left\{G_{A}: A \in \mathfrak{F}_{n}\right\}$ by conjugation. Furthermore, two such groups are in the same orbit if and only if the corresponding Beauville surfaces are isomorphic. This is because any isomorphism $\phi: S_{A}^{n} \longrightarrow S_{A^{\prime}}^{n}$ lifts to an automorphism $\Phi$ of the 
product of curves, yielding a commutative diagram

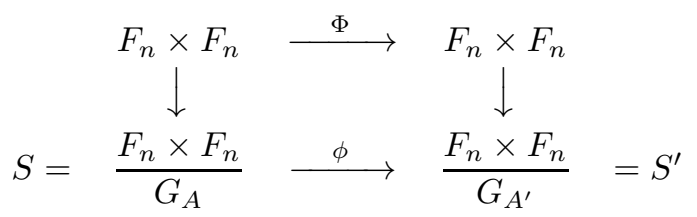

such that $\Phi G_{A} \Phi^{-1}=G_{A^{\prime}}$ (see 6]).

It is well known that the automorphism group of $F_{n}$ is $\mathbb{Z}_{n}^{2} \rtimes \mathcal{S}_{3}$ and so $\operatorname{Aut}\left(F_{n} \times F_{n}\right)=\left\langle\mathbb{Z}_{n}^{2} \times \mathbb{Z}_{n}^{2}, \mathcal{S}_{3} \times \mathcal{S}_{3}, J\right\rangle$, where $J\left(\mu_{1}, \mu_{2}\right)=\left(\mu_{2}, \mu_{1}\right)$ and the groups $\mathbb{Z}_{n}^{2} \times \mathbb{Z}_{n}^{2}$ and $\mathcal{S}_{3} \times \mathcal{S}_{3}$ act separately on each factor, the first one by multiplying the homogeneous coordinates by $n$-th roots of unity and the second one by permuting them. We now note that every element of $\mathbb{Z}_{n}^{2} \times \mathbb{Z}_{n}^{2}$ fixes each element $e_{(\alpha, \beta)}^{A} \in G_{A}$ by conjugation, so the action of this subgroup on the set $\left\{G_{A}\right\}$ is trivial. We can therefore restrict our attention to the quotient group $W=\operatorname{Aut}\left(F_{n} \times F_{n}\right) /\left(\mathbb{Z}_{n}^{2} \times \mathbb{Z}_{n}^{2}\right)$, which is a semidirect product $\left(\mathcal{S}_{3} \times \mathcal{S}_{3}\right) \rtimes\langle J\rangle$ of $\mathcal{S}_{3} \times \mathcal{S}_{3}$ by $\langle J\rangle \cong \mathcal{S}_{2}$, with the complement $\mathcal{S}_{2}$ transposing the direct two factors $\mathcal{S}_{3}$ by conjugation. Thus $W$ is the wreath product $\mathcal{S}_{3}$ 々 $\mathcal{S}_{2}$ of $\mathcal{S}_{3}$ by $\mathcal{S}_{2}$.

The group $\mathcal{S}_{3}$ can be viewed as a subgroup of $\mathrm{GL}_{2}\left(\mathbb{Z}_{n}\right)$ via the group monomorphism:

$$
\begin{array}{cccc}
M: \mathcal{S}_{3} & \longrightarrow & \mathrm{GL}_{2}\left(\mathbb{Z}_{n}\right) \\
\tau & \longmapsto & M_{\tau}
\end{array}
$$

determined by $M_{\sigma_{1}}=\left(\begin{array}{ll}-1 & 1 \\ -1 & 0\end{array}\right)$ for $\sigma_{1}=(1,3,2)$ and $M_{\sigma_{2}}=\left(\begin{array}{ll}0 & 1 \\ 1 & 0\end{array}\right)$ for $\sigma_{2}=(1,2)$.

Lemma 2. Let $\Phi=\left(\tau_{1}, \tau_{2}\right) \in \mathcal{S}_{3} \times \mathcal{S}_{3}$ be a factor-preserving automorphism. Then $\Phi G_{A} \Phi^{-1}=G_{A^{\prime}}$, where $A^{\prime}=M_{\tau_{2}} A M_{\tau_{1}}^{-1}$.

On the other hand, if $\Phi^{\prime}=\Phi \circ J$ is an automorphism interchanging factors, then $\Phi^{\prime} G_{A} \Phi^{\prime-1}=G_{A^{\prime}}$, where $A^{\prime}=M_{\tau_{2}} A^{-1} M_{\tau_{1}}^{-1}$.

Proof. First we note that if $A \in \mathfrak{F}_{n}$, then $A^{\prime}=M_{\tau_{2}} A^{ \pm 1} M_{\tau_{1}}^{-1}$ belongs to $\mathfrak{F}_{n}$ as well. We will now see how the group $\operatorname{Aut}\left(F_{n} \times F_{n}\right)$ acts by conjugation on the elements of $G_{A}$.

To see how an element $\left(\tau_{1}, \tau_{2}\right) \in \mathcal{S}_{3} \times \mathcal{S}_{3}$ acts on $G_{A}$ we will write $\left(\tau_{1}, \tau_{2}\right)=$ $\left(\tau_{1}, \mathrm{Id}\right) \circ\left(\mathrm{Id}, \tau_{2}\right)$, with $\tau_{1}, \tau_{2} \in \mathcal{S}_{3}$. If $\Phi=\left(\mathrm{Id}, \sigma_{1}\right)$ then

$$
\Phi e_{(\alpha, \beta)}^{A} \Phi^{-1}\left(\left[\begin{array}{l}
x_{1} \\
y_{1} \\
z_{1}
\end{array}\right],\left[\begin{array}{c}
x_{2} \\
y_{2} \\
z_{2}
\end{array}\right]\right)=\left(\left[\begin{array}{c}
\xi^{\alpha} x_{1} \\
\xi^{\beta} y_{1} \\
z_{1}
\end{array}\right],\left[\begin{array}{c}
\xi^{(c-a) \alpha+(d-b) \beta} x_{2} \\
\xi^{-a \alpha-b \beta} y_{2} \\
z_{2}
\end{array}\right]\right)
$$

so $\Phi e_{(\alpha, \beta)}^{A} \Phi^{-1}=e_{(\alpha, \beta)}^{A^{\prime}}$ with $A^{\prime}=\left(\begin{array}{rr}c-a & d-b \\ -a & -b\end{array}\right)=M_{\sigma_{1}} A$. In the same way we have that for $\Phi=\left(\mathrm{Id}, \sigma_{2}\right)$, conjugation yields

$$
\Phi e_{(\alpha, \beta)}^{A} \Phi^{-1}\left(\left[\begin{array}{l}
x_{1} \\
y_{1} \\
z_{1}
\end{array}\right],\left[\begin{array}{l}
x_{2} \\
y_{2} \\
z_{2}
\end{array}\right]\right)=\left(\left[\begin{array}{c}
\xi^{\alpha} x_{1} \\
\xi^{\beta} y_{1} \\
z_{1}
\end{array}\right],\left[\begin{array}{c}
\xi^{c \alpha+d \beta} x_{2} \\
\xi^{a \alpha+b \beta} y_{2} \\
z_{2}
\end{array}\right]\right) .
$$


Thus $\Phi e_{(\alpha, \beta)}^{A} \Phi^{-1}=e_{(\alpha, \beta)}^{A^{\prime}}$ again, where $A^{\prime}=\left(\begin{array}{cc}c & d \\ a & b\end{array}\right)=M_{\sigma_{2}} A$ in this case.

Hence conjugation by elements of the form (Id, $\left.\tau_{2}\right)$ acts by left multiplication of the corresponding matrix on $A$.

Now taking $\Phi=\left(\sigma_{1}, \mathrm{Id}\right)$ and conjugating an element of $G_{A}$ we have

$$
\Phi e_{(\alpha, \beta)}^{A} \Phi^{-1}\left(\left[\begin{array}{l}
x_{1} \\
y_{1} \\
z_{1}
\end{array}\right],\left[\begin{array}{l}
x_{2} \\
y_{2} \\
z_{2}
\end{array}\right]\right)=\left(\left[\begin{array}{c}
\xi^{\beta-\alpha} x_{1} \\
\xi^{-\alpha} y_{1} \\
z_{1}
\end{array}\right],\left[\begin{array}{c}
\xi^{a \alpha+b \beta} x_{2} \\
\xi^{c \alpha+d \beta} y_{2} \\
z_{2}
\end{array}\right]\right) .
$$

Thus $\Phi e_{(\alpha, \beta)}^{A} \Phi^{-1}=e_{(\beta-\alpha,-\alpha)}^{A^{\prime}}$ where $A^{\prime}=\left(\begin{array}{cc}b & -a-b \\ d & -c-d\end{array}\right)=A M_{\sigma_{1}}^{-1}$.

Analogously one gets that for $\Phi=\left(\sigma_{2}, \mathrm{Id}\right)$

$$
\Phi e_{(\alpha, \beta)}^{A} \Phi^{-1}\left(\left[\begin{array}{l}
x_{1} \\
y_{1} \\
z_{1}
\end{array}\right],\left[\begin{array}{c}
x_{2} \\
y_{2} \\
z_{2}
\end{array}\right]\right)=\left(\left[\begin{array}{c}
\xi^{\beta} x_{1} \\
\xi^{\alpha} y_{1} \\
z_{1}
\end{array}\right],\left[\begin{array}{c}
\xi^{a \alpha+b \beta} x_{2} \\
\xi^{c \alpha+d \beta} y_{2} \\
z_{2}
\end{array}\right]\right),
$$

and therefore $\Phi e_{(\alpha, \beta)}^{A} \Phi^{-1}=e_{(\beta, \alpha)}^{A^{\prime}}$ where $A^{\prime}=\left(\begin{array}{cc}b & a \\ d & c\end{array}\right)=A M_{\sigma_{2}}^{-1}$.

Finally, for the automorphism $\Phi=J$ we have

$$
\Phi e_{(\alpha, \beta)}^{A} \Phi^{-1}\left(\left[\begin{array}{l}
x_{1} \\
y_{1} \\
z_{1}
\end{array}\right],\left[\begin{array}{c}
x_{2} \\
y_{2} \\
z_{2}
\end{array}\right]\right)=\left(\left[\begin{array}{c}
\xi^{a \alpha+b \beta} x_{1} \\
\xi^{c \alpha+d \beta} y_{1} \\
z_{1}
\end{array}\right],\left[\begin{array}{c}
\xi^{\alpha} x_{2} \\
\xi^{\beta} y_{2} \\
z_{2}
\end{array}\right]\right),
$$

which means that $\Phi e_{(\alpha, \beta)}^{A} \Phi^{-1}=e_{(a \alpha+b \beta, c \alpha+d \beta)}^{A^{-1}}$.

Corollary 2. Two Beauville surfaces $S_{A}^{n}$ and $S_{B}^{n}$ are isomorphic if and only if $B=M_{\tau_{2}} A^{ \pm 1} M_{\tau_{1}}$ for some $\left(\tau_{1}, \tau_{2}\right) \in \mathcal{S}_{3} \times \mathcal{S}_{3}$.

As noted above, $\left|\mathfrak{F}_{5}\right|=24$. One can explicitly write down the 24 matrices and check by hand that these matrices lie in a single orbit under the action of $\operatorname{Aut}\left(F_{n} \times F_{n}\right)$. In this way one obtains the following result:

Corollary 3. Up to isomorphism there is only one Beauville surface with group $\mathbb{Z}_{5}^{2}$, namely $S_{A}^{5}$ where

$$
A=\left(\begin{array}{ll}
1 & 3 \\
2 & 4
\end{array}\right)
$$

This result will also follow from the general formula for the number of isomorphism classes of Beauville surfaces with abelian group given in Theorem 2 .

Remark 2. In [1] Bauer and Catanese state that there are two (non isomorphic) Beauville structures on the product $F_{5} \times F_{5}$ originally considered by Beauville. It seems that this discrepancy is due to the fact that they regard two Beauville surfaces as equivalent if there is a factor-preserving isomorphism between them, whereas here we also consider factor-interchanging isomorphisms such as $J$.

In the next section we will discuss the number of isomorphism classes of Beauville surfaces $S_{A}^{n}$ for each $n$. 


\section{Isomorphism classes of Beauville surfaces with abelian group}

We are interested in finding the number of isomorphism classes of Beauville surfaces with group $\mathbb{Z}_{n}^{2}$ for a given $n$. By Corollary 2 this is equivalent to counting the number of orbits of the group $W=\left(\mathcal{S}_{3} \times \mathcal{S}_{3}\right) \rtimes\langle J\rangle=\mathcal{S}_{3} \succ \mathcal{S}_{2}$ on the set $\mathfrak{F}_{n}$, so by the Cauchy-Frobenius Lemma we deduce that the number of isomorphism classes of Beauville surfaces is

$$
\frac{1}{|W|} \sum_{i}\left|\mathcal{C}_{i}\right|\left|\operatorname{Fix}\left(x_{i}\right)\right|
$$

where $i$ indexes the conjugacy classes $\mathcal{C}_{i}$ of $W$ and $x_{i}$ is any element of $\mathcal{C}_{i}$. The conjugacy classes of $W$ are shown in Table 1, where $\sigma_{2}$ and $\sigma_{3}$ are elements of order 2 and 3 in $S_{3}$.

\begin{tabular}{|c|c|c|c|}
\hline $\begin{array}{c}\text { Conjugacy } \\
\text { class }\end{array}$ & Representative & Order & $\begin{array}{c}\text { Number of } \\
\text { elements }\end{array}$ \\
\hline \hline 1 & $(\mathrm{Id}, \mathrm{Id})$ & 1 & 1 \\
2 & $\left(\mathrm{Id}, \sigma_{2}\right)$ & 2 & 6 \\
3 & $\left(\sigma_{2}, \sigma_{2}\right)$ & 2 & 9 \\
4 & $\left(\mathrm{Id}, \sigma_{3}\right)$ & 3 & 4 \\
5 & $\left(\sigma_{3}, \sigma_{3}\right)$ & 3 & 4 \\
6 & $\left(\sigma_{2}, \sigma_{3}\right)$ & 6 & 12 \\
7 & $(\mathrm{Id}, \mathrm{Id}) \cdot J$ & 2 & 6 \\
8 & $\left(\mathrm{Id}, \sigma_{2}\right) \cdot J$ & 4 & 18 \\
9 & $\left(\sigma_{2}, \sigma_{3} \sigma_{2}\right) \cdot J$ & 6 & 12 \\
\hline
\end{tabular}

Table 1: Conjugacy classes of $W$.

First we need to know the cardinality of the set $\mathfrak{F}_{n}$.

Lemma 3. Let $n$ be a natural number. Then

$$
\left|\mathfrak{F}_{n}\right|=n^{4} \prod_{p \mid n}\left(1-\frac{1}{p}\right)\left(1-\frac{2}{p}\right)\left(1-\frac{3}{p}\right)\left(1-\frac{4}{p}\right)
$$

where $p$ ranges over the distinct primes dividing $n$.

Proof. We will first prove the formula for $n=p$ prime. We want to count the number of matrices $A=\left(\begin{array}{ll}a & b \\ c & d\end{array}\right)$ satisfying condition (1).

Based on the correspondence between matrices $A \in \mathfrak{F}_{n}$ and pairs of triples $((1,0),(0,1),(-1,-1)),((a, c),(b, d),(-a-b,-c-d))$, we deduce that counting matrices in $\mathfrak{F}_{n}$ is equivalent to counting second triples.

Now, any triple in $G$ projects to an ordered triple of points in the projective line $P=\mathbb{P}^{1}\left(\mathbb{Z}_{p}\right)$ formed by the 1 -dimensional subspaces of the vector space 
$G$, so Beauville structures in $G$ correspond to disjoint pairs of triples in $P$. Conversely, any triple in $P$ is induced by $p-1$ triples in $G$, all scalar multiples of each other. The standard triple in $G$ induces the triple $(0, \infty, 1) \in P$. Having chosen the standard triple as the first triple in $G$, there are $|P|-3=p-2$ points of $P$ remaining, so there are $(p-2)(p-3)(p-4)$ choices for the second triple in $P$, corresponding to $(p-1)(p-2)(p-3)(p-4)$ triples in $G$.

The case $n=p^{e}$ follows from the following fact. Having fixed the standard triple as the first one, any second triple given by $a, b, c \in \mathbb{Z}_{p^{e}}^{2}$ satisfying (i)-(iii) will give by reduction modulo $p$ a triple $(a \bmod p, b \bmod p, c \bmod p)$. Conversely, for each triple $(a, b, c)$ with $a, b, c \in \mathbb{Z}_{p}^{2}$, each of the $p^{4 e-4}$ choices for $\mathrm{v}=$ $\left(h_{1}, h_{2}, j_{1}, j_{2}\right)$ with $h_{1}, h_{2}, j_{1}, j_{2}=0, \ldots, p^{e-1}-1$ gives a triple $\left(a_{\mathrm{v}}, b_{\mathrm{v}}, c_{\mathrm{v}}\right)$ where

$$
\begin{aligned}
& a_{\mathrm{v}}=a+\left(h_{1} p, h_{2} p\right) \\
& c_{\mathrm{v}}=c+\left(j_{1} p, j_{2} p\right) \\
& b_{\mathrm{v}}=-a_{\mathrm{v}}-c_{\mathrm{v}}
\end{aligned}
$$

such that $\left(a_{\mathrm{v}} \bmod p, b_{\mathrm{v}} \bmod p, c_{\mathrm{v}} \bmod p\right)=(a, b, c)$.

Finally, we can extend the formula to all $n$ by a straightforward application of the Chinese Remainder Theorem.

We note that our formula for $\left|\mathfrak{F}_{n}\right|$ is equivalent to that given by Garion and Penegini in [12, Corollary 3.24] for the function denoted there by $N_{n}$.

Lemma 3 immediately gives us information about the asymptotic behaviour of $\left|\mathfrak{F}_{n}\right|$ for large $n$. If $n=p^{e}$ for some prime $p \geq 5$ then

$$
\frac{\left|\mathfrak{F}_{n}\right|}{n^{4}}=\left(1-\frac{1}{p}\right)\left(1-\frac{2}{p}\right)\left(1-\frac{3}{p}\right)\left(1-\frac{4}{p}\right) \geq \frac{4 !}{5^{4}},
$$

and $\left|\mathfrak{F}_{n}\right| / n^{4} \rightarrow 1$ as $p \rightarrow \infty$. However, if $n$ is divisible by the first $k$ primes $p_{i} \geq 5$ then since

$$
\lim _{k \rightarrow \infty} \prod_{i}\left(1-\frac{1}{p_{i}}\right)=0
$$

(see Exercise 9.3 of [15]) we have $\left|\mathfrak{F}_{n}\right| / n^{4} \rightarrow 0$ as $k \rightarrow \infty$.

Since the isomorphism classes of Beauville surfaces with Beauville group $\mathbb{Z}_{n}^{2}$ correspond to the orbits of $W$ on $\mathfrak{F}_{n}$, and $|W|=72$, there are at least $\left|\mathfrak{F}_{n}\right| / 72$ such classes. We will now apply the Cauchy-Frobenius Lemma to find the exact number of isomorphism classes. For this, we need to calculate how many matrices in $\mathfrak{F}_{n}$ are fixed by each element of the group.

First of all we note that an element of $W$ fixes a matrix in $\mathfrak{F}_{n}$ if and only if it fixes its components modulo the prime powers $p_{i}^{e_{i}}$ in the factorisation of $n$, so we can again restrict our attention to prime powers.

It is obvious that $x_{1}=(\mathrm{Id}, \mathrm{Id})$ fixes every matrix in $\mathfrak{F}_{n}$, so the number of its fixed points is

$$
\Theta_{1}(n):=\left|\operatorname{Fix}\left(x_{1}\right)\right|=\left|\mathfrak{F}_{n}\right| .
$$


On the other hand it is easy to see that the conditions on a matrix $A \in \mathrm{GL}_{2}\left(\mathbb{Z}_{n}\right)$ to be fixed by an element of the conjugacy classes $\mathcal{C}_{2}, \mathcal{C}_{3}, \mathcal{C}_{4}, \mathcal{C}_{6}$ or $\mathcal{C}_{8}$ are incompatible with those defining $\mathfrak{F}_{n}$.

The action of $x_{5}=\left(\sigma_{3}, \sigma_{3}\right) \in \mathcal{C}_{5}$ sends a matrix $A=\left(\begin{array}{ll}a & b \\ c & d\end{array}\right)$ to $\left(\sigma_{3}, \sigma_{3}\right) A=$ $\left(\begin{array}{c}d-b(a+b)-(c+d) \\ -b\end{array}\right)$. In the case of $n=p^{e}$ a prime power, the conditions $c=-b$ and $d=a+b$ leave $p^{2 e}(1-1 / p)(1-2 / p)$ possibilities for the matrix $A=$ $\left(\begin{array}{cc}a & b \\ -b & a+b\end{array}\right)$, from which we have to remove those with a non-unit determinant. If $p \equiv-1 \bmod 3$ then the equation $a^{2}+a b+b^{2} \equiv 0 \bmod p$ has no solutions, since it is equivalent to $\lambda^{2}+\lambda+1 \equiv 0$ and $\mathbb{F}_{p}$ contains no non-trivial third root of unity. Otherwise, for a fixed $a$ there are two non-valid choices for $b$. Therefore

$$
\Theta_{2}\left(p^{e}\right):=\left|\operatorname{Fix}\left(x_{5}\right)\right|= \begin{cases}p^{2 e}\left(1-\frac{1}{p}\right)\left(1-\frac{2}{p}\right), & \text { if } p \equiv-1 \bmod 3 \\ p^{2 e}\left(1-\frac{1}{p}\right)\left(1-\frac{4}{p}\right), & \text { if } p \equiv 1 \bmod 3 .\end{cases}
$$

For $x_{7}=(\mathrm{Id}, \mathrm{Id}) \cdot J \in \mathcal{C}_{7}$, the action on $A$ yields (Id, Id) $J(A)=A^{-1}$. The equality $A=A^{-1}$ in $\mathfrak{F}_{p^{e}}$ implies that $a=-d$ and $b(\operatorname{det}(A)+1)=0$ and hence $\operatorname{det}(A)=-1$. The general form for a matrix fixed by this element is therefore $A=\left(\begin{array}{cc}a & b \\ c & -a\end{array}\right)$, with $\operatorname{det}(A)=-a^{2}-b c=-1$. There are $p^{e}(1-3 / p)$ possibilities for $a \not \equiv 0, \pm 1 \bmod p$, and once $a$ is chosen there are $p^{e}(1-5 / p)$ choices left for $b \not \equiv 0,-a, \frac{1-a^{2}}{a}, 1-a,-1-a \bmod p$. The final formula gives

$$
\Theta_{3}\left(p^{e}\right):=\left|\operatorname{Fix}\left(x_{7}\right)\right|=p^{2 e}(1-3 / p)(1-5 / p)
$$

Finally $x_{9}=\left(\sigma_{2}, \sigma_{3} \sigma_{2}\right) \cdot J \in \mathcal{C}_{9}$ acts by sending $A$ to $\left(\sigma_{2}, \sigma_{3} \sigma_{2}\right) J(A)=$ $\left(\begin{array}{ccc}-c & c-a \\ d & b & -d\end{array}\right) / \operatorname{det}(A)$. The conditions imply that $\operatorname{det}(A)$ is $\operatorname{different}$ from -1 and $\operatorname{det}(A)=\operatorname{det}(A)^{3}$, hence $\operatorname{det}(A)=1$. Writing down the rest of the conditions one gets $a=-c=-d$ and $b=-2 a$, so we are looking for elements $a \in \mathbb{Z}_{p^{e}}$ such that $-3 a^{2} \equiv 1 \bmod p^{e}$. Now -3 is a square in $\mathbb{Z}_{p^{e}}$ if and only if it is a quadratic residue modulo $p$ (see e.g. Thm. 7.14 in [15]), and this is the case only when $p \equiv 1 \bmod 3$. To see this note that if $p \equiv \varepsilon \bmod 3$, where $\varepsilon= \pm 1$, by the Law of Quadratic Reciprocity

$$
\left(\frac{3}{p}\right)= \begin{cases}\varepsilon, & \text { if } p \equiv 1 \bmod 4 \\ -\varepsilon, & \text { if } p \equiv 3 \bmod 4\end{cases}
$$

Therefore $\left(\frac{-3}{p}\right)=\left(\frac{-1}{p}\right)\left(\frac{3}{p}\right)=\varepsilon$. As a consequence

$$
\Theta_{4}\left(p^{e}\right):=\left|\operatorname{Fix}\left(x_{9}\right)\right|= \begin{cases}0, & \text { if } p \equiv-1 \bmod 3 ; \\ 2, & \text { if } p \equiv 1 \bmod 3 .\end{cases}
$$

We have thus proved the following:

Theorem 2. Let $n=p_{1}^{e_{1}} \cdot \ldots \cdot p_{s}^{e_{s}}$ be a natural number coprime to 6, where $p_{1}, \ldots, p_{k}$ are distinct primes. Then the number of isomorphism classes of Beauville surfaces with Beauville group $\mathbb{Z}_{n}^{2}$ is

$$
\Theta(n)=\frac{1}{72}\left(\Theta_{1}(n)+4 \prod_{i=1}^{s} \Theta_{2}\left(p_{i}^{e_{i}}\right)+6 \prod_{i=1}^{s} \Theta_{3}\left(p_{i}^{e_{i}}\right)+12 \prod_{i=1}^{s} \Theta_{4}\left(p_{i}^{e_{i}}\right)\right),
$$


where the functions $\Theta_{i}$ are defined as above.

The formulae given above for $\Theta_{r}\left(p^{e}\right)$ for $r=2,3$ and 4 show that the sum on the right-hand side of this equation is dominated by the first term, so that

$$
\Theta(n) \sim \frac{1}{72} \Theta_{1}(n)=\frac{1}{72}\left|\mathfrak{F}_{n}\right|=\frac{n^{4}}{72} \prod_{p \mid n}\left(1-\frac{1}{p}\right)\left(1-\frac{2}{p}\right)\left(1-\frac{3}{p}\right)\left(1-\frac{4}{p}\right)
$$

as $n \rightarrow \infty$. In particular, we have the following special case:

Corollary 4. For each prime $p \geq 5$ the number $\Theta\left(p^{e}\right)$ of isomorphism classes of Beauville surfaces with Beauville group $\mathbb{Z}_{p^{e}}^{2}$ is given by

$$
\begin{array}{r}
\frac{1}{72}\left(p^{4 e}-10 p^{4 e-1}+35 p^{4 e-2}-50 p^{4 e-3}+24 p^{4 e-4}+10 p^{2 e}-60 p^{2 e-1}\right. \\
\left.+98 p^{2 e-2}\right)
\end{array}
$$

if $p \equiv-1 \bmod (3)$, and by

$$
\begin{aligned}
\frac{1}{72}\left(p^{4 e}-10 p^{4 e-1}+35 p^{4 e-2}-50 p^{4 e-3}+24 p^{4 e-4}+10 p^{2 e}\right. & -68 p^{2 e-1} \\
& \left.+106 p^{2 e-2}+24\right)
\end{aligned}
$$

if $p \equiv 1 \bmod (3)$.

When $e=1$ this specialises to

$$
\Theta(p)= \begin{cases}\frac{1}{72}\left(p^{4}-10 p^{3}+45 p^{2}-110 p+122\right), & \text { if } p \equiv-1 \bmod 3 \\ \frac{1}{72}\left(p^{4}-10 p^{3}+45 p^{2}-118 p+154\right), & \text { if } p \equiv 1 \bmod 3 .\end{cases}
$$

Remark 3. In 2], Bauer, Catanese and Grunewald considered the asymptotic behaviour of the number of Beauville surfaces with Beauville group $\mathbb{Z}_{n}^{2}$, where $n$ is coprime to 6 . Later, Garion and Penegini 12 considered a wide range of related counting problems; in particular, they obtained bounds similar to those in 2] for Beauville groups $G=\mathbb{Z}_{n}^{2}$, specifically that $\Theta(n)$ lies between $\left|\mathfrak{F}_{n}\right| / 72$ and $\left|\mathfrak{F}_{n}\right| / 6$ (Corollary 3.24). Their results are consistent with ours.

\section{The automorphism group of $S_{A}^{n}$}

The calculations in the previous section provide some insight into the automorphism group of a Beauville surface with an abelian Beauville group.

The fact that the automorphisms of $S_{A}^{n}$ lift to automorphisms of $F_{n} \times F_{n}$ shows that all automorphisms of $S_{A}^{n}$ are induced by elements of $N\left(G_{A}\right)$, the normaliser of $G_{A}$ in $\operatorname{Aut}\left(F_{n} \times F_{n}\right)$ and, in fact, that $\operatorname{Aut}\left(S_{A}^{n}\right) \cong N\left(G_{A}\right) / G_{A}$. 
Since clearly

$$
G_{A} \leq \mathbb{Z}_{n}^{2} \times \mathbb{Z}_{n}^{2} \leq N\left(G_{A}\right)
$$

one sees immediately that each surface $S_{A}^{n}$ admits the group $\left(\mathbb{Z}_{n}^{2} \times \mathbb{Z}_{n}^{2}\right) / G_{A}$ as a group of automorphisms. Moreover, one can identify the Beauville group $\mathbb{Z}_{n}^{2}$ with $\left(\mathbb{Z}_{n}^{2} \times \mathbb{Z}_{n}^{2}\right) / G_{A}$ by sending a pair $(\alpha, \beta)$ to $((0,0),(\alpha, \beta)) \bmod G_{A}$, which acts as an automorphism of $S_{A}^{n}$ by multiplying the coordinates of the second factor by the corresponding roots of unity. Actually this simply reflects the known fact (see [14]) that a Beauville surface with group $G$ always has the centre $Z(G)$ of $G$ as a group of automorphisms.

This isomorphism $\mathbb{Z}_{n}^{2} \cong\left(\mathbb{Z}_{n}^{2} \times \mathbb{Z}_{n}^{2}\right) / G_{A}$ allows a further identification

$$
\operatorname{Aut}\left(S_{A}^{n}\right) / \mathbb{Z}_{n}^{2}=N\left(G_{A}\right) /\left(\mathbb{Z}_{n}^{2} \times \mathbb{Z}_{n}^{2}\right),
$$

where the group on the right is simply the stabiliser in $\operatorname{Aut}\left(F_{n} \times F_{n}\right) /\left(\mathbb{Z}_{n}^{2} \times \mathbb{Z}_{n}^{2}\right)=$ $W$ of the matrix $A$.

In fact we have the following:

Proposition 1. Let $H$ be the subgroup of $W$ identified as above with the quotient $\operatorname{Aut}\left(S_{A}^{n}\right) / \mathbb{Z}_{n}^{2}$. Then $H \cong\{\operatorname{Id}\}, \mathbb{Z}_{2}, \mathbb{Z}_{3}, \mathbb{Z}_{6}$ or $\mathcal{S}_{3}$.

Proof. By the comment above, an element of $W$ will induce an automorphism of $S_{A}^{n}$, and therefore belong to $H$, if and only if it fixes the matrix $A$. By the proof of Theorem 2, the only elements with fixed points in $\mathfrak{F}_{n}$ are those in the conjugacy classes $\mathcal{C}_{1}, \mathcal{C}_{5}, \mathcal{C}_{7}$ and $\mathcal{C}_{9}$, so $H \subseteq \mathcal{C}_{1} \cup \mathcal{C}_{5} \cup \mathcal{C}_{7} \cup \mathcal{C}_{9}$.

Let $H^{0}=H \cap\left(\mathcal{S}_{3} \times \mathcal{S}_{3}\right)$, so that $\left|H: H^{0}\right| \leq 2$ and $H^{0} \subseteq \mathcal{C}_{1} \cup \mathcal{C}_{5}$. If $\gamma_{1}, \gamma_{2} \in \mathcal{C}_{5}$ and $\gamma_{2} \neq \gamma_{1}^{ \pm 1}$ then $\gamma_{1} \gamma_{2} \in \mathcal{C}_{4}$, so $\gamma_{1} \gamma_{2} \notin H$; it follows that $\left|H^{0}\right|=1$ or 3 , and hence $|H|=1,2,3$ or 6 . The only groups of these orders are those listed in the Proposition.

Moreover, by the discussion prior to Theorem 2 and the formula for $\Theta_{4}\left(p^{e}\right)$, if $n$ is divisible by some prime $p \equiv-1 \bmod 3$ then no element of order 6 in $W$ fixes points of $\mathfrak{F}_{n}$. Similarly, the formula for $\Theta_{3}\left(p^{e}\right)$, together with the fact that no element of $\mathcal{C}_{2} \cup \mathcal{C}_{3}$ has fixed points, ensures that no element of order 2 can belong to $H$ if 5 divides $n$. We therefore have the following:

Corollary 5. Let $S_{A}^{n}$ be a Beauville surface. If a prime $p \equiv-1 \bmod 3$ divides $n$, then $H \cong\{\operatorname{Id}\}, \mathbb{Z}_{2}, \mathbb{Z}_{3}$ or $\mathcal{S}_{3}$. If 5 divides $n$ then $H \cong\{\operatorname{Id}\}$ or $\mathbb{Z}_{3}$.

For instance, in Beauville's original example [5], Aut $\left(S_{A}^{5}\right)$ is a semidirect product of $\mathbb{Z}_{5}^{2}$ by $H \cong \mathbb{Z}_{3}$.

This analysis of automorphism groups has been extended to more general Beauville surfaces in [14].

Acknowledgement. The first and third author authors were partially supported by MEC grant MTM2009-11848. The third author was partially supported by an FPU grant of the MICINN. 


\section{References}

[1] Bauer, I., Catanese, F.: Some new surfaces with $p_{g}=q=0$, Proceedings of the Fano Conference Torino, 123-142 (2004).

[2] Bauer, I., Catanese, F., Grunewald, F.: Beauville surfaces without real structures I, Geometric methods in algebra and number theory, 1-42 (2005).

[3] Bauer, I., Catanese, F.: A volume maximizing canonical surface in 3-space, Comment. Math. Helv. 83, no. 2, 387-406 (2008).

[4] Bauer, I., Catanese, F., Grunewald, F.: The classification of surfaces with $p_{g}=q=0$ isogenous to a product of curves, Pure Appl. Math. Q. 4, no. 2, part 1, 547-586 (2008).

[5] Beauville, A.: Surfaces algébriques complexes. Astérisque, No. 54. Société Mathématique de France, Paris (1978).

[6] Catanese, F.: Fibred surfaces, varieties isogenous to a product and related moduli spaces, Amer. J. Math. 122, 1-44 (2000).

[7] Fairbairn, B., Magaard, K., Parker, C.: Generation of finite simple groups with an application to groups acting on Beauville surfaces. arXiv:1010.3500 (2010).

[8] Fuertes, Y., González-Diez, G.: On Beauville structures on the groups $S_{n}$ and $A_{n}$, Math. Z. 264, no. 4 , 959-968 (2010).

[9] Fuertes, Y., González-Diez, G., Jaikin, A.: On Beauville surfaces, Groups Geom. Dyn. 5, no. 1, 107-119 (2011).

[10] Fuertes, Y., Jones, G. A.: Beauville structures and finite groups, J. Algebra 340, 13-27 (2011).

[11] Garion, S., Larsen, M., Lubotzky, A.: Beauville surfaces and finite simple groups. arXiv:1005.2316 (2010).

[12] Garion, S., Penegini, M.: Beauville surfaces, moduli spaces and finite groups. arXiv:1107.5534v1 (2011).

[13] Guralnick, R., Malle, G.: Simple groups admit Beauville structures. arXiv:1009.6183 (2010).

[14] Jones, G. A.: Automorphism groups of Beauville surfaces. arXiv:1102.3055 (2011).

[15] Jones, G. A., Jones, J. M.: Elementary Number Theory. Springer Undergraduate Mathematics Series. Springer-Verlag London, Ltd., London (1998). 
G. GonZÁlez-Diez:

Departamento de Matemáticas, Universidad Autónoma de Madrid, 28049, Madrid, Spain.

email: gabino.gonzalez@uam.es

G. A. Jones:

School of Mathematics, University of Southampton, Southampton SO17 1BJ, U.K.

email: G.A.Jones@maths.soton.ac.uk

D. TORRES-TeigelL:

Departamento de Matemáticas, Universidad Autónoma de Madrid, 28049, Madrid, Spain. email: david.torres@uam.es 